\title{
Radiofrequency Catheter Ablation: How to Manage and Prevent Collateral Damage?
}

\author{
MOHAMMAD EL BABA, MD, ${ }^{1}$ DEAN SABAYON, MD², and \\ MARWAN M. REFAAT, MD, FACC, FAHA, FHRS, FASE, FESC, FACP, FRCP ${ }^{3,4}$
}

${ }^{1}$ Electrophysiology Section, University Hospitals Cleveland Medical Center, Cleveland, OH, USA

${ }^{2}$ Electrophysiology Section, Emory University Hospital, Atlanta, GA, USA

${ }^{3}$ Department of Internal Medicine, Division of Cardiology/Electrophysiology Section, American University of Beirut Medical Center, Beirut, Lebanon

${ }^{4}$ Department of Biochemistry and Molecular Genetics, American University of Beirut Faculty of Medicine, Beirut, Lebanon

\begin{abstract}
Radiofrequency catheter ablation has become the standard of care for the management of various arrhythmias and, in fact, the first-line therapy for many tachyarrhythmias. It entails creating scar tissue in the heart in regions where abnormal impulses form or propagate to restore normal cardiac conduction. As the heart is a complex organ and is surrounded by and related to many other anatomical structures, it is important to avoid the collateral damage that can happen from radiofrequency $(R F)$ ablation on the endocardium as well as on the epicardium. This review explores methods for mitigating or limiting collateral damage during catheter ablation.
\end{abstract}

KEYWORDS. Ablation, atrial fibrillation, collateral damage, management, ventricular tachycardia.

\begin{abstract}
ISSN 2156-3977 (print) ISSN 2156-3993 (online) CC BY 4.0 license
\end{abstract}

(c) 2020 Innovations in Cardiac Rhythm Management

\section{Introduction}

Radiofrequency (RF) catheter ablation has become the standard treatment for various arrhythmias and is the first-line therapy for treating many tachyarrhythmias. ${ }^{1}$ RF ablation adopts a high-frequency $(500-750 \mathrm{~Hz})$ current to produce lesions through the process of thermal injury. Resistive heating damages the tissue in direct contact with the ablation catheter. Deeper tissues are heated and damaged by heat conduction. ${ }^{2}$ To cause tissue necrosis, the temperature at the electrode-tissue interface must be

The authors report no conflicts of interest for the published content. Manuscript received October 24, 2019. Final version accepted May 4, 2020.

Address correspondence to: Marwan M. Refaat, MD, FACC, FAHA, FHRS, FASE, FESC, FACP, FRCP, Department of Internal Medicine, Cardiovascular Medicine/Cardiac Electrophysiology, Department of Biochemistry and Molecular Genetics, American University of Beirut Faculty of Medicine and Medical Center, PO Box 11-0236, Riad El-Solh, Beirut 1107 2020, Lebanon. Email: mr48@aub.edu.lb. at least $50^{\circ} \mathrm{C}$ but, at temperatures approaching $100^{\circ} \mathrm{C}$, a coagulum of denatured proteins forms on the catheter tip, limiting the delivery of current and increasing the risk of thromboembolic complications. ${ }^{3}$

As the heart is a complex organ and is surrounded by and related to many other structures, it is important to avoid any collateral damage linked to RF ablation on both the endocardium and the epicardium.

Complications vary depending on the procedure but serious complications are rare (eg, death, myocardial infarction, or stroke occurring in $0.05 \%-0.01 \%$ ), with a higher risk of stroke correlated with atrial fibrillation (AF) ablation. ${ }^{4}$ Heart block requiring a permanent pacemaker is rare and occurs in $0.5 \%$ of catheter ablations. Vascular access complications occur in $2 \%$ to $4 \%$ of cases and cardiac tamponade occurs in $1 \%$ to $2 \%$ of cases. Phrenic nerve $(\mathrm{PN})$ damage may be found especially after $\mathrm{AF}$ ablation. Also, with AF ablation, pulmonary vein (PV) stenosis may be observed. Meanwhile, atrioesophageal fistulae are extremely rare. 
In this review, the complications and types of collateral damage from RF ablation of various arrhythmias as well as the measures to avoid them will be discussed.

\section{Prevention and management of complications and collateral damage in atrial fibrillation ablation}

$\mathrm{AF}$ is the most common sustained arrhythmia, with significant morbidity and mortality rates. PV isolation has been reported to be an effective strategy for the treatment of symptomatic AF. However, despite developments in the technology and improvements in the techniques, catheter ablation for AF remains a highly complex procedure with a non-negligible risk of complications. Major complications have been reported to appear in up to $5.2 \%$ of procedures, ${ }^{5-7}$ while death occurs in one out of every 1,000 cases. $^{8}$ In a systematic review by Cappato et al., the causes of deaths in 32,569 patients included tamponade in eight patients (including one case later than 30 days postprocedure), stroke in five patients (including two cases later than 30 days postprocedure), atrioesophageal fistula in five patients, and massive pneumonia in two patients. Myocardial infarction, intractable torsade de pointes, septicemia, sudden respiratory arrest, extrapericardial PV perforation, occlusion of both lateral PVs, hemothorax, and anaphylaxis were reported to be responsible for one death each, while asphyxia from tracheal compression secondary to subclavian hematoma, intracranial bleeding, acute respiratory distress syndrome, and esophageal perforation from an intraoperative transesophageal echocardiographic probe were causes of one late death each, respectively. ${ }^{8}$

Pulmonary vein stenosis. The PVs have been demonstrated to be substantial in AF initiation and maintenance. ${ }^{9,10}$ Around $88.8 \%$ of ectopic beats initiating $\mathrm{AF}$ originate from the PVs. PV isolation is now performed in $\mathrm{AF}$ ablation procedures.

During the early work with AF ablation and before the improvement of clinical experience, isolation of the PVs was carried out by applying RF lesions within the veins or at the venoatrial junction. Thus, PV stenosis has been a major concern after AF ablation. Early reports showed an incidence of PV stenosis of between $8 \%$ and $15 \% .{ }^{11-13}$ Factors contributing to the development of PV stenosis included the application of energy inside the veins and inappropriate energy delivery. ${ }^{12,13}$ It remains unclear exactly how PV stenosis develops. Responses to thermal injury within the PVs, which include intimal proliferation with organizing thrombus, necrotic myocardium in various stages of collagen replacement, endovascular contraction, and the proliferation of elastic lamina, could be a potential explanation. ${ }^{14}$

The most common symptom resulting from severe PV stenosis is dyspnea on exertion. Also, dyspnea at rest, recurrent cough, pleuritic chest pain, flu-like symptoms, and hemoptysis have been reported. ${ }^{15}$ Patients with symptomatic PV stenosis are often diagnosed late due to the nonspecific nature of their symptoms. ${ }^{16}$
The location and extent of the stenosis are best evaluated by computed tomography (CT). Lung perfusion scans also can be beneficial, especially to determine the effect of the stenosis. Symptoms of PV stenosis develop when the perfusion of the affected lobe falls below $20 \%$ or the perfusion of the entire lung on the affected side falls below $25 \%{ }^{17}$

PV angioplasty remains the best treatment option for symptomatic PV stenosis. In a study by Prieto et al., stent angioplasty resulted in less restenosis than dilation, particularly for stents measuring $10 \mathrm{~mm}$ or larger. ${ }^{17}$ Here, the restenosis rates were $72 \%$ for dilation versus $33 \%$ for stenting $(p<0.001)$ over a mean follow-up period of 25 months. Time to restenosis was greater for stent angioplasty $(p=0.003)$. Conversely, the management of asymptomatic patients is challenging and risks should be weighed with the potential benefits of the procedure. $^{12}$

Overall, the incidence of severe PV stenosis is decreasing. In one report, the incidence rate of severe PV stenosis was less than $1 \%$ and the incidence rate of symptomatic PV stenosis necessitating intervention was negligible. ${ }^{18}$ This is mainly due to the change in the ablation technique by adopting an antral ablation approach. ${ }^{19}$ The wide diameter of the antrum prevents PV stenosis, even in the presence of scar retraction at the ablation sites. The adoption of this new ablation technique has drastically decreased symptomatic PV stenosis. ${ }^{12}$

However, some areas of the PV, such as the ridge that separates the left PVs from the left atrial (LA) appendage, make it impossible to conduct PV ablation from a distance. In addition, the esophagus sometimes lies in close proximity to the PVs on one side and ablation must be performed on the PV itself to avoid damaging the esophagus.

Atrioesophageal fistula. Atrioesophageal fistula is as an extremely rare $(0.04 \%)$ but catastrophic complication of the catheter ablation of $\mathrm{AF}^{20}$

The esophagus lies posterior to the LA with a variable course relative to the LA adjacent to the right or left PVs or the posterior wall. ${ }^{21,22}$ Therefore, there is a risk of esophageal damage when RF energy is delivered anywhere in the LA, including particularly in the left atrium posterior wall. The nonuniform thickness of the posterior LA wall and the variable fibrofatty layer between the wall and the esophagus are risk factors that should be kept in mind during the ablation procedure. Esophageal arteries and the vagus nerve plexus on the anterior surface of the esophagus can also be affected by ablative procedures. Patients with AF and LA dilatation generally present a larger LA-esophageal contact area and thinner fat pads. Once esophageal necrosis develops, mediastinitis and fistula occur, connecting the esophageal lumen with the pericardium. It has also been reported that gastroesophageal reflux may facilitate mucosal injury, thus playing an additional role. ${ }^{23}$ 
Potential strategies to prevent esophageal injury include: (1) empirically reducing the power and duration of energy application on the posterior LA wall, (2) avoiding overlapping ablation lines, (3) monitoring esophageal temperature, (4) monitoring the esophageal position in relation to the posterior LA, and (5) educating the patient regarding signs and symptoms of esophageal injury.

Endoscopic evaluation postablation reveals a high $(15 \%-48 \%)$ rate of incidence of esophageal erosion in patients undergoing RF for PV isolation. ${ }^{24}$ Predictors of esophageal injury and the potential for the development of esophageal fistula ${ }^{24}$ include persistent AF ablation, power of greater than $30 \mathrm{~W}$, an increase in esophageal temperature to higher than $40^{\circ} \mathrm{C}$, intraoperative pain (if sedation is used), and proximity of the esophagus to the LA wall.

Pericardial effusion and cardiac tamponade. The occurrence of cardiac tamponade following AF ablation is rare, ${ }^{25}$ with an incidence rate of $0.98 \%$ per procedure and $1.46 \%$ per patient. It is the most frequent cause of death occurring in association with AF ablation. ${ }^{8}$ In particular, its incidence is higher when conducting AF ablation $(0.8 \%-2.9 \%) .6,26,27$ This can be attributed to the complexity of the procedure, frequent manipulation with catheters, extensive ablation, and systemic anticoagulation.

Cardiac perforation leading to cardiac tamponade can occur (1) during transseptal puncture (puncture of the right atrial posterior wall or puncture the roof, appendage, or lateral LA wall); (2) during catheter manipulation (tear of the LA appendage or roof of the LA); or (3) during the delivery of RF energy (overheating with the development of steam pop, leading to myocardial rupture).

Clinically, cardiac tamponade presents as an abrupt fall in blood pressure. The use of intracardiac echocardiography (ICE) allows for the earlier detection of pericardial effusion ${ }^{28}$ and reduces the risk of cardiac tamponade. ${ }^{29}$

The majority of patients who develop cardiac tamponade can be managed by percutaneous drainage, but some may require surgical closure. The LA roof is susceptible to perforation that may not be responsive to pericardiocentesis. On the roof, the pericardium is not adherent to the LA. ${ }^{28}$

Steam pop due to tissue boiling related to the delivery of high levels of energy is a risk factor of cardiac tamponade. Limiting the energy delivered can significantly decrease the incidence of cardiac tamponade. ${ }^{27}$

Stroke and thromboembolic complications. Thromboembolism is one of the most serious and feared complications of AF ablation. The incidence of periprocedural thromboembolic ranges from $1 \%$ to $7 \%{ }^{7,8}$ Silent cerebral ischemia detected on magnetic resonance imaging is substantial, with risk factors including the level of activated clotting time and cardioversion to sinus rhythm during the procedure. ${ }^{30}$

The risk of cerebral thromboembolism is at its highest in the first two weeks following ablation, with the most likely cause being the formation of char and/or thrombus at the sites of ablation.

Preprocedural management of anticoagulants. The continuation of anticoagulation with warfarin reduces the risk of periprocedural stroke without increased bleeding complications. ${ }^{31,32} \mathrm{Di}$ Biase et al. demonstrated that the combination of an open-irrigation ablation catheter and periprocedural therapeutic anticoagulation using warfarin may, in fact, reduce the risk of periprocedural stroke without increasing the risk of bleeding or pericardial effusion.

Novel oral anticoagulants (NOACs) can also be continued during the procedure. Di Biase et al. showed that an uninterrupted apixaban strategy is effective in preventing thromboembolic events without increasing the bleeding risk, ${ }^{33}$ while Cappato et al. suggested that the use of uninterrupted oral rivaroxaban is feasible, with a similar event rate to that of uninterrupted warfarin. ${ }^{34}$ Further, in a study by Calkins et al., anticoagulation with uninterrupted dabigatran was associated with less bleeding than that seen with uninterrupted warfarin. ${ }^{35}$

Phrenic nerve injury. PN paralysis has been described after RF ablation and is a rare complication occurring in $0.48 \%$ of patients. ${ }^{36}$ Ablation near the right superior PV or within the superior vena cava can affect the right $\mathrm{PN} .^{36}$ Complete and partial recovery were observed in $66 \%$ and $17 \%$ of patients, respectively. Symptoms include dyspnea, hiccups, cough, and pain, while pleural effusion and atelectasis can also be seen. Fluoroscopy confirms the diagnosis.

To prevent PN injury, a number of strategies have been designed. These include ensuring antral PV ablation; high-output pacing to establish whether the PN can be captured from the proposed ablation site before ablation; PN mapping with anatomic tagging of its course using an electroanatomic mapping system; and monitoring of the diaphragmatic excursion with abdominal palpation, fluoroscopy, or intracardiac ultrasound while pacing the PN from the superior vena cava or subclavian vein during ablation. ${ }^{37}$

Direct monitoring of diaphragmatic compound motor action potentials (CMAPs) during ablation using diaphragmatic electromyography is another technique that has been suggested to further reduce the incidence of PN injury. ${ }^{38,39}$ CMAPs are recorded using body surface electrodes, esophageal electrodes, or a diagnostic catheter positioned in the hepatic vein. In some studies, a decrease in the amplitude of the myopotential by $30 \%$ has been reported to be more sensitive than abdominal palpation for predicting the subsequent reduction in diaphragmatic excursion and nerve injury. ${ }^{40}$ 
Vagal nerve plexus injury. RF delivery to the posterior wall of the LA may cause injury to the vagal esophageal plexus, leading to pyloric spasm, gastric hypomotility, and a markedly prolonged gastric-emptying time. ${ }^{41}$ In the study by Shah et al., four of 367 patients who underwent AF ablation developed symptoms of vagal nerve injury within 48 hours after the procedure. ${ }^{41}$ Symptoms included abdominal bloating and discomfort hours to days after the procedure. ${ }^{42,43}$ Spontaneous recovery may require a time frame of up to one year. Prevention involves conducting lower-power and shorter-duration ablation on the posterior wall.

\section{Prevention and management of complications and collateral damage in epicardial ventricular tachycardia ablation}

Ventricular tachycardia (VT) is a challenging arrhythmia for both patients and physicians alike to deal with. Antiarrhythmic medications have limited effectiveness and are often poorly tolerated. ${ }^{44,45}$ Meanwhile, catheter ablation is being adopted increasingly more frequently and appears to be reasonably effective. ${ }^{46-48} \mathrm{In}$ 2012 , a total of 2,927 VT ablation procedures were performed in the United States. ${ }^{49}$ Most VTs can be ablated endocardially but some require epicardial mapping and ablation.

Bleeding. Hemopericardium is seen with pericardial access in about $8 \%$ of patients. ${ }^{50}$ Right ventricular puncture/laceration, coronary vessel puncture/laceration, and/or adhesion disruption are common reasons for hemopericardium. Double right-ventricular perforation could lead to extensive bleeding when the sheath is removed at the end of the case.

ICE plays an important role in identifying pericardial effusion, including being able to identify the wire if it is in the RV. Most cases of bleeding with RV punctures from the access needle are self-limited if the sheath is not advanced into the RV. Similarly, most small-vessel punctures or adhesion disruptions stop bleeding without intervention other than aspiration. However, the occurrence of major-vessel puncture or chamber laceration requires cardiac surgery to address.

Phrenic nerve. The left PN should be identified by high output pacing, ranging from 20 to $50 \mathrm{~mA}$ with a 2-ms pulse width. ${ }^{51}$ When ablation needs to be performed adjacent to the PN, it can be displaced. ${ }^{52}$ Di Biase et al. demonstrated that controlled and progressive inflation of air and saline with careful monitoring of hemodynamic parameters appears to be the best strategy for preventing PN injury during epicardial ablation. A balloon catheter can also be used to separate the PN from the ablation catheter. $^{53}$

As mentioned previously, monitoring of diaphragmatic excursion with abdominal palpation, fluoroscopy, or intracardiac ultrasound while pacing the PN from the superior vena cava or subclavian vein during ablation ${ }^{37}$ is another technique that can be used to prevent PN injury.

Epicardial coronary arteries. Coronary arteries can be damaged by RF ablation. ${ }^{54}$ A coronary angiogram is routinely performed to define the relative location of the coronary arteries relative to the area of interest and establishing a distance of at least $5 \mathrm{~mm}$ away during this procedure from the coronary arteries is recommended. The adoption of multiple angiographic views should be completed to assess the distance appropriately.

\section{Prevention and management of complications and collateral damage in supraventricular tachycardia ablation}

SVT ablation is performed to treat arrhythmias involving the upper chambers of the heart. This procedure aims to reduce symptoms of tachycardia and improve the patient's quality of life. Once inside the heart, the catheters are used to stimulate and record electrical signals to induce the clinical arrhythmia, understand its mechanism of action, and identify the treatment site that would yield the best result for termination of the tachyarrhythmia. The success rate of an SVT ablation ranges between 93 and $97 \%$ 55,56 and its associated complication rate is $0.8 \%{ }^{5}$

The major complications from this procedure are conduction system damage and cardiac perforation. In particular, inadvertent damage to the AV node or His bundle is the most feared complication during an SVT ablation. This phenomenon most commonly occurs in patients requiring slow-pathway modification for the treatment of atrioventricular nodal reentry tachycardia (AVNRT) because of the close proximity of the AV node to the anatomic area of the slow pathway. The slow pathway is usually located in the posteroinferior aspect of the triangle of Koch, while the AV node and His bundle are located in the anterosuperior aspect of this triangle. These anatomic landmarks can be blurred and not clearly well-demarcated in some patients, rendering slow-pathway modification particularly challenging. This occurs especially in young patients due to the vertical orientation of their hearts or elderly patients due to the posterior rotation of their hearts. The incidence rate of complete heart block in patients while attempting slow-pathway modification for treatment of AVNRT is $0.8 \% .{ }^{.5}$

More recent data have suggested an increased risk of late pacemaker implantation after AVNRT ablation. After AVNRT ablation, the risk of late pacemaker implantation was low but remained three times higher than that in patients without AVNRT and three times higher than the risk of periprocedural pacemaker implantation. ${ }^{58}$ Furthermore, ablation does not seem to be the cause of the heightened late pacemaker implantation risk. 
There is a very minimal risk of inadvertent heart block in patients being treated by catheter ablation for typical atrial flutter because of the relatively far distance of the cavotricuspid isthmus to the AV node and His bundle. The same holds true for lateral wall accessory pathways. However, the risk is much higher for para-Hisian accessory pathways. The risk of inadvertent complete heart block while attempting a para-Hisian accessory pathway ablation is $3 \%$ to $5 \% .{ }^{59-61}$

Cryoablation may carry a lower risk of AV blockage, but this mode of therapy is associated with a higher recurrence rate. ${ }^{62-64}$

\section{Conclusion}

In summary, here, we reviewed the types of collateral damage associated with RF ablation of AF, VT, and SVT. We also discussed the types of collateral damage associated with each type of ablation procedure and the prevention measures available in each context.

It is very important to have a good understanding of the anatomy during RF ablation-not only what is in contact with the ablation catheter but also what is around the area being ablated. This can help both to enhance procedural outcomes and limit or avoid complications.

\section{References}

1. Guidelines for Clinical Intracardiac Electrophysiological and Catheter Ablation Procedures. A report of the American College of Cardiology/American Heart Association Task Force on Practice Guidelines (Committee on Clinical Intracardiac Electrophysiologic and Catheter Ablation Procedures). Developed in collaboration with the North American Society of Pacing and Electrophysiology. Circulation. 1995;92(3):673-691.

2. Nath S, DiMarco JP, Haines DE. Basic aspects of radiofrequency catheter ablation. I Cardiovasc Electrophysiol. 1994;5(10):863-876.

3. Michelucci A, Antonucci E, Conti AA, et al. Electrophysiologic procedures and activation of the hemostatic system. Am Heart J. 1999;138(1):128-132.

4. Chen S-A, Chiang C-E, Tai C-T, et al. Complications of diagnostic electrophysiologic studies and radiofrequency catheter ablation in patients with tachyarrhythmias: an eight-year survey of 3,966 consecutive procedures in a tertiary referral center. Am J Cardiol. 1996;77(1):41-46.

5. Bohnen M, Stevenson WG, Tedrow UB, et al. Incidence and predictors of major complications from contemporary catheter ablation to treat cardiac arrhythmias. Heart Rhythm. 2011;8(11):1661-1666.

6. Cappato R, Calkins H, Chen S-A, et al. Worldwide survey on the methods, efficacy, and safety of catheter ablation for human atrial fibrillation. Circulation. 2005;111(9):1100-1105.

7. Cappato R, Calkins H, Chen S-A, et al. Updated worldwide survey on the methods, efficacy, and safety of catheter ablation for human atrial fibrillation. Circ Arrhythm Electrophysiol. 2010;3(1):32-38.

8. Cappato R, Calkins H, Chen S-A, et al. Prevalence and causes of fatal outcome in catheter ablation of atrial fibrillation. J Am Coll Cardiol. 2009;53(19):1798-1803.
9. Chen SA, Hsieh MH, Tai CT, et al. Initiation of atrial fibrillation by ectopic beats originating from the pulmonary veins: electrophysiological characteristics, pharmacological responses, and effects of radiofrequency ablation. Circulation. 1999;100(18):1879-1886.

10. Jaïs P, Hocini M, Macle L, et al. Distinctive electrophysiological properties of pulmonary veins in patients with atrial fibrillation. Circulation. 2002;106(19):2479-2485.

11. Gerstenfeld EP, Guerra P, Sparks PB, Hattori K, Lesh MD. Clinical outcome after radiofrequency catheter ablation of focal atrial fibrillation triggers. J Cardiovasc Electrophysiol. 2001;12(8):900-908.

12. Saad EB, Rossillo A, Saad CP, et al. Pulmonary vein stenosis after radiofrequency ablation of atrial fibrillation: functional characterization, evolution, and influence of the ablation strategy. Circulation. 2003;108(25):3102-3107.

13. Yu W-C, Hsu T-L, Tai C-T, et al. Acquired pulmonary vein stenosis after radiofrequency catheter ablation of paroxysmal atrial fibrillation. J Cardiovasc Electrophysiol. 2001;12(8):887-892.

14. Taylor GW, Kay GN, Zheng X, Bishop S, Ideker RE. Pathological effects of extensive radiofrequency energy applications in the pulmonary veins in dogs. Circulation. 2000;101(14):1736-1742.

15. Packer DL, Keelan P, Munger TM, et al. Clinical presentation, investigation, and management of pulmonary vein stenosis complicating ablation for atrial fibrillation. Circulation. 2005;111(5):546-554.

16. Saad EB, Marrouche NF, Saad CP, et al. Pulmonary vein stenosis after catheter ablation of atrial fibrillation: emergence of a new clinical syndrome. Ann Intern Med. 2003;138(8):634-638.

17. Prieto LR, Schoenhagen P, Arruda MJ, Natale A, Worley SE. Comparison of stent versus balloon angioplasty for pulmonary vein stenosis complicating pulmonary vein isolation. $J$ Cardiovasc Electrophysiol. 2008;19(7):673-678.

18. Teunissen C, Velthuis BK, Hassink RJ, et al. Incidence of pulmonary vein stenosis after radiofrequency catheter ablation of atrial fibrillation. JACC Clin Electrophysiol. 2017;3(6):589-598.

19. Ouyang F, Bänsch D, Ernst S, et al. Complete isolation of left atrium surrounding the pulmonary veins: new insights from the double-lasso technique in paroxysmal atrial fibrillation. Circulation. 2004;110(15):2090-2096.

20. Martinek M, Bencsik G, Aichinger J, et al. Esophageal damage during radiofrequency ablation of atrial fibrillation: impact of energy settings, lesion sets, and esophageal visualization. J Cardiovasc Electrophysiol. 2009;20(7):726-733.

21. Sánchez-Quintana D, Cabrera JA, Climent V, Farré J, de Mendonça MC, Ho SY. Anatomic relations between the esophagus and left atrium and relevance for ablation of atrial fibrillation. Circulation. 2005;112(10):1400-1405.

22. Thiagalingam A, Manzke R, D'Avila A, et al. Intraprocedural volume imaging of the left atrium and pulmonary veins with rotational X-ray angiography: implications for catheter ablation of atrial fibrillation. J Cardiovasc Electrophysiol. 2008;19(3):293-300.

23. Martinek M, Hassanein S, Bencsik G, et al. Acute development of gastroesophageal reflux after radiofrequency catheter ablation of atrial fibrillation. Heart Rhythm. 2009;6(10):1457-1462.

24. Martinek M, Meyer C, Hassanein S, et al. Identification of a high-risk population for esophageal injury during radiofrequency catheter ablation of atrial fibrillation: procedural and anatomical considerations. Heart Rhythm. 2010;7(9):1224-1230. 
25. Hamaya R, Miyazaki S, Taniguchi H, et al. Management of cardiac tamponade in catheter ablation of atrial fibrillation: single-centre 15 year experience on 5222 procedures. Europace. 2018;20(11):1776-1782.

26. Bertaglia E, Zoppo F, Tondo C, et al. Early complications of pulmonary vein catheter ablation for atrial fibrillation: a multicenter prospective registry on procedural safety. Heart Rhythm. 2007;4(10):1265-1271.

27. Hsu L-F, Jaïs P, Hocini M, et al. Incidence and prevention of cardiac tamponade complicating ablation for atrial fibrillation. Pacing Clin Electrophysiol. 2005;28(s1):S106-S109.

28. Bunch TJ, Asirvatham SJ, Friedman PA, et al. Outcomes after cardiac perforation during radiofrequency ablation of the atrium. J Cardiovasc Electrophysiol. 2005;16(11): $1172-1179$.

29. Aldhoon B, Wichterle D, Peichl P, Čihák R, Kautzner J. Complications of catheter ablation for atrial fibrillation in a high-volume centre with the use of intracardiac echocardiography. Europace. 2013;15(1):24-32.

30. Gaita F, Caponi D, Pianelli M, et al. Radiofrequency catheter ablation of atrial fibrillation: a cause of silent thromboembolism?: magnetic resonance imaging assessment of cerebral thromboembolism in patients undergoing ablation of atrial fibrillation. Circulation. 2010;122(17):1667-1673.

31. Di Biase L, Burkhardt JD, Mohanty P, et al. Periprocedural stroke and management of major bleeding complications in patients undergoing catheter ablation of atrial fibrillation: the impact of periprocedural therapeutic international normalized ratio. Circulation. 2010;121(23):2550-2556.

32. Santangeli P, Di Biase L, Horton R, et al. Ablation of atrial fibrillation under therapeutic warfarin reduces periprocedural complications: evidence from a meta-analysis. Circ Arrhythm Electrophysiol. 2012;5(2):302-311.

33. Di Biase L, Lakkireddy D, Trivedi C, et al. Feasibility and safety of uninterrupted periprocedural apixaban administration in patients undergoing radiofrequency catheter ablation for atrial fibrillation: results from a multicenter study. Heart Rhythm. 2015;12(6):1162-1168.

34. Cappato R, Marchlinski FE, Hohnloser SH, et al. Uninterrupted rivaroxaban vs. uninterrupted vitamin $\mathrm{K}$ antagonists for catheter ablation in non-valvular atrial fibrillation. Eur Heart J. 2015;36(28):1805-1811.

35. Calkins H, Willems S, Gerstenfeld EP, et al. Uninterrupted dabigatran versus warfarin for ablation in atrial fibrillation. N Engl J Med. 2017;376(17):1627-1636.

36. Sacher F, Monahan KH, Thomas SP, et al. Phrenic nerve injury after atrial fibrillation catheter ablation: characterization and outcome in a multicenter study. J Am Coll Cardiol. 2006;47(12):2498-2503.

37. Yong Ji S, Dewire J, Barcelon B, et al. Phrenic nerve injury: an underrecognized and potentially preventable complication of pulmonary vein isolation using a wide-area circumferential ablation approach: phrenic nerve injury. J Cardiovasc Electrophysiol. 2013;24(10):1086-1091.

38. Miyazaki S, Hachiya $\mathrm{H}$, Taniguchi $\mathrm{H}$, et al. Prospective evaluation of bilateral diaphragmatic electromyograms during cryoballoon ablation of atrial fibrillation: bilateral diaphragmatic CMAPs during cryoablation. I Cardiovasc Electrophysiol. 2015;26(6):622-628.

39. Franceschi F, Dubuc M, Guerra PG, Khairy P. Phrenic nerve monitoring with diaphragmatic electromyography during cryoballoon ablation for atrial fibrillation: the first human application. Heart Rhythm. 2011;8(7):1068-1071.

40. Mondésert B, Andrade JG, Khairy P, et al. Clinical experience with a novel electromyographic approach to preventing phrenic nerve injury during cryoballoon ablation in atrial fibrillation. Circ Arrhythm Electrophysiol. 2014;7(4): 605-611.

41. Shah D, Dumonceau J-M, Burri H, et al. Acute pyloric spasm and gastric hypomotility: an extracardiac adverse effect of percutaneous radiofrequency ablation for atrial fibrillation. J Am Coll Cardiol. 2005;46(2):327-330.

42. Pisani CF, Hachul D, Sosa E, Scanavacca M. Gastric hypomotility following epicardial vagal denervation ablation to treat atrial fibrillation. I Cardiovasc Electrophysiol. 2008;19(2):211-213.

43. Bunch TJ, Ellenbogen KA, Packer DL, Asirvatham SJ. Vagus nerve injury after posterior atrial radiofrequency ablation. Heart Rhythm. 2008;5(9):1327-1330.

44. Connolly SJ. Comparison of $\beta$-blockers, amiodarone plus $\beta$-blockers, or sotalol for prevention of shocks from implantable cardioverter defibrillators the OPTIC study: a randomized trial. JAMA. 2006;295(2):165-171.

45. Deyell MW, Steinberg C, Doucette S, et al. Mexiletine or catheter ablation after amiodarone failure in the VANISH trial. J Cardiovasc Electrophysiol. 2018;29(4):603-608.

46. Muser D, Santangeli P, Castro SA, et al. Long-term outcome after catheter ablation of ventricular tachycardia in patients with nonischemic dilated cardiomyopathy. Circ Arrhythm Electrophysiol. 2016;9(10): pii: e004328.

47. Tung R, Vaseghi M, Frankel DS, et al. Freedom from recurrent ventricular tachycardia after catheter ablation is associated with improved survival in patients with structural heart disease: an International VT Ablation Center Collaborative Group study. Heart Rhythm. 2015;12(9):1997-2007.

48. Sapp JL, Wells GA, Parkash R, et al. Ventricular tachycardia ablation versus escalation of antiarrhythmic drugs. $N$ Engl J Med. 2016;375(2):111-121.

49. Yousuf OK, Zusterzeel R, Sanders W, et al. Trends and outcomes of catheter ablation for ventricular tachycardia in a community cohort. JACC Clin Electrophysiol. 2018;4(9):1189-1199.

50. Tung R, Michowitz Y, Yu R, et al. Epicardial ablation of ventricular tachycardia: an institutional experience of safety and efficacy. Heart Rhythm. 2013;10(4):490-498.

51. Fan R, Cano O, Ho SY, et al. Characterization of the phrenic nerve course within the epicardial substrate of patients with nonischemic cardiomyopathy and ventricular tachycardia. Heart Rhythm. 2009;6(1):59-64.

52. Di Biase L, Burkhardt JD, Pelargonio G, et al. Prevention of phrenic nerve injury during epicardial ablation: comparison of methods for separating the phrenic nerve from the epicardial surface. Heart Rhythm. 2009;6(7):957-961.

53. Buch E, Vaseghi M, Cesario DA, Shivkumar K. A novel method for preventing phrenic nerve injury during catheter ablation. Heart Rhythm. 2007;4(1):95-98.

54. D'Avila A, Gutierrez P, Scanavacca M, et al. Effects of radiofrequency pulses delivered in the vicinity of the coronary arteries: implications for nonsurgical transthoracic epicardial catheter ablation to treat ventricular tachycardia. Pacing Clin Electrophysiol. 2002;25(10):1488-1495.

55. Calkins H, Yong P, Miller JM, et al. Catheter ablation of accessory pathways, atrioventricular nodal reentrant tachycardia, and the atrioventricular junction: final results of a prospective, multicenter clinical trial. Circulation. 1999;99(2):262-270.

56. Spector P, Reynolds MR, Calkins H, et al. Meta-Analysis of Ablation of Atrial Flutter and Supraventricular Tachycardia. Am J Cardiol. 2009;104(5):671-677.

57. Scheinman MM, Huang S. The 1998 NASPE prospective catheter ablation registry. Pacing Clin Electro. 2000;23(6):1020-1028. 
58. Kesek M, Lindmark D, Rashid A, Jensen SM. Increased risk of late pacemaker implantation after ablation for atrioventricular nodal reentry tachycardia: a 10-year follow-up of a nationwide cohort. Heart Rhythm. 2019;16(8):1182-1188.

59. Gatzoulis K, Apostolopoulos T, Costeas X, et al. Paraseptal accessory connections in the proximity of the atrioventricular node and the His bundle. Additional observations in relation to the ablation technique in a high risk area. Europace. 2004;6(1):1-9.

60. Mandapati R, Berul CI, Triedman JK, Alexander ME, Walsh EP. Radiofrequency catheter ablation of septal accessory pathways in the pediatric age group. Am J Cardiol. 2003;92(8):947-950.

61. Van Hare GF, Javitz H, Carmelli D, et al. Prospective assessment after pediatric cardiac ablation: demographics, medical profiles, and initial outcomes. J Cardiovasc Electrophysiol. 2004;15(7):759-770.

62. Matta M, Anselmino M, Scaglione M, et al. Cooling dynamics: a new predictor of long-term efficacy of atrioventricular nodal reentrant tachycardia cryoablation. J Interv Card Electrophysiol. 2017;48(3):333-341.

63. Hanninen M, Yeung-Lai-Wah N, Massel D, et al. Cryoablation versus RF ablation for AVNRT: a meta-analysis and systematic review: meta-analysis of cryoablation for AVNRT. J Cardiovasc Electrophysiol. 2013;24(12):1354-1360.

64. Deisenhofer I, Zrenner B, Yin Y, et al. Cryoablation versus radiofrequency energy for the ablation of atrioventricular nodal reentrant tachycardia (the CYRANO Study): results from a large multicenter prospective randomized trial. Circulation. 2010;122(22):2239-2245. 